\title{
ICDs in nonischaemic heart failure
}

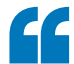

the decidedly negative results of DANISH should not surprise us
The use of an implantable cardioverter-defibrillator (ICD) in patients with systolic heart failure not caused by coronary artery disease does not reduce all-cause mortality. This finding comes from the DANISH trial, which was presented at the 2016 ESC Congress in Rome, Italy, and simultaneously published in The New England Journal of Medicine. The trial "will change the way cardiologists think about ICD use in patients with heart failure," says John Mandrola (Louisville Cardiology Group, Kentucky, USA), in a commentary published on Medscape.

Prophylactic use of an ICD in patients with heart failure and reduced ejection fraction has a class 1 recommendation in both the European and US guidelines. However, the evidence for this recommendation is much stronger for patients with ischaemic heart disease than for those with heart failure from other causes.

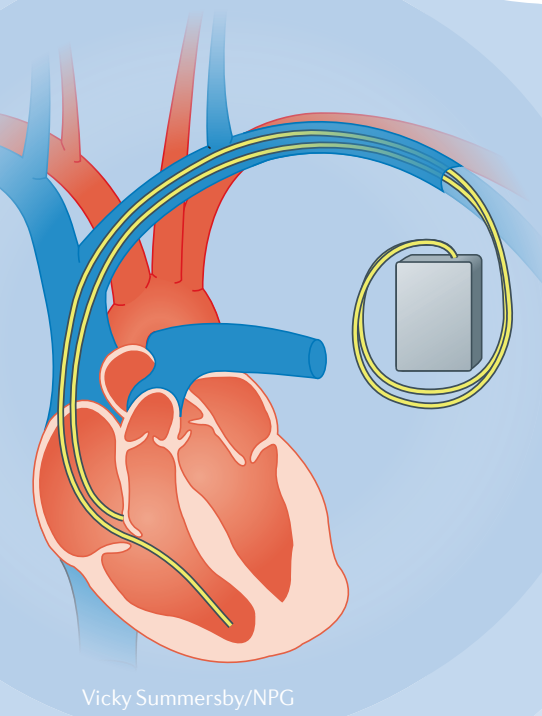

To assess the use of ICDs in patients with nonischaemic heart failure, the DANISH investigators randomly assigned 1,116 patients with stable, chronic, symptomatic, nonischaemic heart failure with a reduced ejection fraction $(\leq 35 \%)$ and an increased level of $\mathrm{N}$-terminal pro-B-type natriuretic peptide ( $>200 \mathrm{pg} / \mathrm{ml}$ ) either to receive or not to receive an ICD, in addition to usual clinical care. Background medical therapy was comprehensive, with almost all patients receiving an angiotensin-converting-enzyme inhibitor or an angiotensin-receptor blocker and a $\beta$-blocker, and nearly $60 \%$ receiving a mineralocorticoidreceptor antagonist. In both groups, $58 \%$ of patients received cardiac resynchronization therapy (CRT).

During follow-up (median 67.6 months), the primary outcome of all-cause mortality was $21.6 \%$ in the ICD group and $23.4 \%$ in the control group (HR 0.87, 95\% CI $0.68-1.12, P=0.28)$. Cardiovascular death also was not significantly different between the two groups (13.8\% vs $17.0 \%$; HR $0.77,95 \%$ CI $0.57-1.05, P=0.10$ ), but sudden cardiac death was significantly reduced with ICD use (4.3\%) compared with the control group (8.2\%; HR 0.50, $95 \%$ CI $0.31-0.82, P=0.005)$. Device infections occurred in $4.9 \%$ of the ICD group and $3.6 \%$ of the control group $(P=0.29)$.

"The results of the DANISH trial," writes John McMurray (University of Glasgow, UK) in an editorial accompanying the trial publication, "probably represent the most optimistic estimate of the benefit of ICD therapy in patients with nonischaemic heart failure who receive evidence-based therapy." Mandrola believes that "the decidedly negative results of DANISH should not surprise us", because patients with nonischaemic heart failure are less prone to arrhythmia than those with ischaemic heart failure, background medical therapy in the trial was robust, and many patients received CRT. Combined, these factors lower mortality, making it harder to demonstrate a benefit of ICD use. "The absolute benefit of ICDs in a typical, well-treated population with heart failure might be small," concludes McMurray.

In subgroup analyses, all-cause mortality was significantly lower among patients aged $<68$ years than among older patients (HR 0.64, 95\% CI 0.45-0.90, $P=0.01$ ), which the investigators believe suggests that "younger patients may have a survival benefit in association with ICD implantation". This finding was independent of CRT.

"ICDs are expensive and not without adverse effects," summarizes McMurray. "It is, therefore, desirable to avoid their use in patients who are unlikely to benefit. These considerations highlight the need to target ICDs to the patients who remain at high absolute risk despite receiving the best available pharmacological and device therapy."

Gregory B. Lim

ORIGINAL ARTICLE Køber, L. et al. Defibrillator implantation in patients with nonischemic systolic heart failure. N. Engl. J. Med. http://dx.doi. org/10.1056/NEJMoa1608029 (2016) FURTHER READING Lewis, G. F. et al. Clinical experience with subcutaneous implantable cardioverter-defibrillators. Nat. Rev. Cardiol. 12, 398-405 (2016) 